Draft VERSION JANUARY 15, 2021

Preprint typeset using $\mathrm{LAT}_{\mathrm{E}} \mathrm{X}$ style emulateapj v. 5/2/11

\title{
EVIDENCE FOR A VERY LOW-COLUMN DENSITY HOLE IN THE GALACTIC HALO IN THE DIRECTION OF THE HIGH LATITUDE MOLECULAR CLOUD MBM 16
}

\author{
W. Liu, M. Galeazzi, and E. URsino \\ Physics Department, University of Miami, Coral Gables, FL 33124, USA; galeazzi@physics.miami.edu \\ Draft version January 15, 2021
}

\begin{abstract}
Shadow observations are the only way to observe emission from the galactic halo $(\mathrm{GH})$ and/or the circumgalactic medium (CGM) free of any foreground contamination from local hot bubble (LHB) and solar wind charge exchange (SWCX). We analyzed data from a shadow observation in the direction of the high latitude, neutral hydrogen cloud MBM 16 with Suzaku. We found that all emission can be accounted for by foreground emission from LHB and SWCX, plus power law emission associated with unresolved point sources. The GH/CGM in the direction of MBM 16 is negligible or inexistent in our observation, with upper limits on the emission measure of $9.5 \times 10^{-4} \mathrm{pc} \mathrm{cm}{ }^{-6}$ (90\% C.L.), at the lowest end of current estimates.

Subject headings: ISM: individual (local hot bubble) — Galaxy: halo - X-rays: diffuse background
\end{abstract}

\section{INTRODUCTION}

Is the Milky Way surrounded by a hot halo a few kpc deep? Or is the X-ray emission we observe from a more extended $(\sim 100 \mathrm{kpc})$ circumgalactic medium $(\mathrm{CGM})$ ? Is this region uniform of clumpy? Is its shape spherical or pancake-like? These are some of the questions about the origin of the absorbed X-ray emitting region at $3 / 4$ $\mathrm{keV}$ observed by ROSAT. A region we know very little about, despite its influence on the evolution of our Galaxy, due to its weak, diffuse emission and its overlap with foreground emission from Solar Wind Charge eXchange (SWCX) and Local Hot Bubble (LHB).

The ROSAT All Sky Survey proved the existence of emission, characterized as plasma at $\sim 3 \times 10^{6} \mathrm{~K}$. ROSAT showed that the emission is of galactic origin (but farther away than the LHB) but could not give any better indication of its location. More recently, higher resolution absorption and emission investigations (i.e., Nicastro et al. 2002; Williams et al. 2007, Galeazzi et al. 2007; Gupta et al. 2009: Henley \& Shelton 2012) detected the presence of $\mathrm{O} V \mathrm{VII}$ and $\mathrm{O} \mathrm{VIII}$ lines at $\mathrm{z} \sim 0$, but the nature of this gas is still disputed. One possible interpretation is that $\mathrm{O}$ VII and O VIII are associated to the predicted WHIM (e.g., Cen \& Ostriker 1999) in the Local Group, thus providing a partial answer to the missing baryons problem. On the other hand, this gas could be located well within the Galactic halo (GH). Distinguishing between the two possibilities is not easy since detectors do not have high enough energy resolution. Following Henley \& Shelton (2012), we can define two models, the GH/CGM is a large shell of gas that envelopes the Milky Way up to $\sim 100 \mathrm{kpc}$, with almost isotropic emission on large scale, or the GH/CGM is gas flowing away from the Galactic Plane, at a distance of $\sim 10 \mathrm{kpc}$. In this case the emission should have radial dependence $I=I_{0} \cos |b|$, where $b$ is the galactic latitude.

Either model does not account for finer details in the $\mathrm{GH} / \mathrm{CGM}$ structure. For example, if the cause of the emission is due to hot gas infalling from our neighborhood, then we would expect a filament-like structure, highly inhomogeneous. On the other hand, if the emis- sion is due to hot gas escaping from the Milky Way, then it is expected to be in the form of fountains, creating a patchy halo on smaller scale.

Henley \& Shelton (2012) analyzed a large number of O VII and O VIII observations performed with XMMNewton at different positions in the sky. Their analysis ruled out the possibility that the gas is at $T \gg 3 \times 10^{6}$ $\mathrm{K}$, but the high variance in the data does not allow to set stricter values on other parameters and to put limits on the GH/CGM models: the temperature of the GH/CGM appears to be uniform $\left(\sim 2 \times 10^{6} \mathrm{~K}\right)$ on all sky surveys, while the corresponding emission measure (EM) spans an order of magnitude (Henley \& Shelton 2013; Yoshino et al. 2009). After filtering the emission line catalog by Henley \& Shelton (2010, 2012), Miller \& Bregman (2015) found a good constraint using O VIII emission lines on the GH density profile, suggesting a galactic plane origin, while the O VII constraint is dominated by temperature or density variations in the LHB. However, the analysis was performed on single pointing observations, giving rise to high uncertainties due to the poorly constrained contribution of the foreground (LHB and SWCX).

Shadow observations allow for the separation of foreground and background emission and are currently the only tool to study the GH/CGM emission without the large systematic uncertainties coming from contamination due to LHB and SWCX.

Shadow observations are performed by looking at a high column density cloud with distance of a few hundred parsecs from the Sun, and at a low density target a few degrees away. Since the cloud is a strong absorber, it shields most of the X-rays emitted by background sources and leaves an almost pure foreground spectrum. The signal toward the low density line of sight, instead, includes also a large fraction of the contribution from distant Xray components. By comparing the spectra in the lines of sight of the cloud and the nearby target, we can characterize the spectral properties of the distant component.

So far very few targets suitable for shadow experiments have been observed with any of the three major X-ray satellites (Chandra, XMM-Newton, and Suzaku). Our group has focused on the neutral hydrogen cloud MBM20 
with XMM-Newton (Galeazzi et al. 2007) and Suzaku (Gupta et al.2009). In addition there are available observations of the neutral hydrogen cloud MBM12 performed with Chandra (Smith et al. 2005), Suzaku (Smith et al. 2007) and XMM-Newton (Koutroumpa et al. 2011), and that of a relatively dense neutral hydrogen filament in the southern galactic hemisphere (Henley \& Shelton 2008). A detailed comparison of the observations is reported in Gupta et al. (2009).

Section 2 of this paper will discuss the data reduction of Suzaku data. Section 3 will focus on the data analysis and results from the Shadow observation, and section 4 will focus on comparison with other cloud observations (MBM12 and MBM20) and the implications of the MBM16 result.

\section{DATA REDUCTION}

The high-column-density molecular cloud MBM16 is part of a Suzaku SWCX key project targets for the characterization of SWCX, and has been observed several times in the last few years. During Suzaku cycle 8 we obtained the observation of a low density region a few degrees away from MBM16, to be performed right after the key project observation of MBM16. The timing of the two observations minimizes the SWCX variation and makes it possible to model the SWCX as a constant component rather than being time variable. The most important parameter for the observations and the analysis are reported in table 1 . In this paper we will usually refer to the two targets as "On-cloud" and "Off-cloud" (or simply "On" and "Off"), meaning MBM16 and the low density target, respectively.

The assessment of the absorption properties is critical for this investigation. Large scale neutral hydrogen surveys (Dickey \& Lockman 1990; Kalberla et al. 2005), however, have very poor angular resolution, of the order of the Suzaku field of view, and cannot resolve the individual parts of the two targets. On the other hand, IRAS $100 \mu \mathrm{m}$ maps have excellent angular resolution and allow for a full characterization of the targets. In order to compute the required hydrogen density, we converted the IRAS $100 \mu \mathrm{m}$ intensity to neutral hydrogen column density $\left(\mathrm{N}_{H}\right)$ using the "typical" high-latitude $100 \mu \mathrm{m} / \mathrm{N}_{H}$ ratio of $0.85 \times 10^{-20} \mathrm{~cm}^{2} \mathrm{MJy} \mathrm{sr}^{-1}$ (Boulanger \& Perault 1988).

The data reduction procedure is relatively standard and we already used it several times for other works (e.g., Mitsuishi et al. 2012), including the analysis of the Suzaku key project data. The raw data are reprocessed with the most update calibration files. The $5 \times 5$ and $3 \times 3$ mode-data are merged to increase the size of the sample. We filtered the data for high flaring and anomalous regions where the satellite is exposed to high particle flux and extracted clean event files and the 0.4-2.0 keV maps of the targets. We identified and removed point sources using wavelet detection for a $120^{\prime \prime}$ PSF. After additional filtering for the cut-off rigidity of the Earth's magnetic field, we extracted the spectra and generated the corresponding exposure maps, response and ancillary files, and spectra of non-X-ray background. The X-ray and non-X-ray spectra are normalized to a uniform $20^{\prime}$ circular field. The non-X-ray spectrum is further rescaled to match the observed spectrum in the $11-14.5 \mathrm{keV}$ range, where the effective area is negligible and the observed signal is only of non-X-ray origin. We did not include data from the XIS0 and XIS3 chips. They have a small effective area at the low energy tail of our interest and give little contribution to the signal.

In recent years a new contaminant is increasingly affecting observations, the $\mathrm{O}$ I line at $0.525 \mathrm{keV}$ (Sekiya et al. 2014) resulting from fluorescence of solar X-rays with neutral $\mathrm{O}$ in the Earth's atmosphere. Lack of proper handling of the O I signal leads to large bias in the estimate of the nearby O VII lines and could lead to wrong interpretation of the properties of the emitting plasma. Rather than excluding time intervals with elevation angle larger than $60^{\circ}$ as suggested by Sekiya et al. (2014) and losing a lot of the available data, we included the O I contamination in our models by adding an emission line at $0.525 \mathrm{keV}$.

\section{DATA ANALYSIS AND RESULTS}

The X-ray emission toward MBM16 On- and Off-cloud should consist of foreground emission (LHB plus SWCX), GH/CGM emission, and extragalactic X-ray background from unresolved point sources. To model the foreground emission (LHB and SWCX), a single apec thermal model has often been used in the past (Foster et al. 2012, Galeazzi et al. 2007; Henley \& Shelton 2008; Gupta et al. 2009). In our analysis, we used an improved model that includes an un-absorbed apec model with $\mathrm{O}$ abundance fixed at 0 , plus three $\delta$-functions at the energies of $\mathrm{O}$ VII line $(0.57 \mathrm{keV})$, and O VIII line $(0.65 \mathrm{keV}$ and $0.81 \mathrm{keV})$ to take into account the variation induced by SWCX. We also included one additional Gaussian line at energy of $0.525 \mathrm{keV}$ to model the O I line as mentioned in Section 2. We modeled the $\mathrm{GH}$ emission as an absorbed apec thermal model, with its temperature and normalization free, and modeled the extragalactic emission with an absorbed power law, where both the index and normalization are free parameters.

In order to separate the foreground and background emission, we fitted the On- and Off-cloud spectra simultaneously, with the model parameters of the On- and Off-cloud tied together except for the absorbing column density. We performed spectral analysis in the energy band 0.4-5.0 keV, using the XSPEC version 12.8 (Arnaud 1996), adopting the metal abundance model by Anders \& Grevesse (1989). Both spectra are re-binned to have at least 50 counts per bin. To verify that the fit had not become trapped in a local minimum, we explored the local parameter space by varying individual parameters over a range centered on the best-fitting values.

In Figure 1 we show the On- and Off-cloud spectra of MBM16 together with the best fitting model, which is decomposed into different model components (foreground in green, $\mathrm{GH}$ in blue and extragalactic component in magenta). In general the fit is good with a reduced $\chi^{2}$ of $0.93\left(\chi^{2} /\right.$ dof $\left.=302.61 / 326\right)$. In table 2 we present the results of spectral modeling (errors are quoted at $90 \%$ confidence). The best fit temperature of the LHB is consistent with previous studies (Snowden et al. 1998 Kuntz \& Snowden 2000; Liu et al. 2015). We also verified that the contamination from O I line to the O VII line is small and excluding the O I component does not affect our results. The temperature of the GH/CGM is consistent with previous estimates Henley \& Shelton 2013), but with large uncertainty. This is because the 
TABLE 1

Observations of MBM 16 On- ANd OfF-ClOud

\begin{tabular}{ccccccc}
\hline \hline Target & Observation ID & $\mathrm{l}(\mathrm{deg})$ & $\mathrm{b}(\mathrm{deg})$ & Start Date & Livetime $(\mathrm{s})$ & $\mathrm{N}_{H}\left(10^{20} \mathrm{~cm}^{-2}\right)$ \\
\hline MBM 16 ON & 5080078010 & 170.58 & -37.28 & $2013-08-07$ & 69053 & 33.2 \\
MBM 16 OFF & 5080073010 & 165.84 & -38.39 & $2013-08-09$ & 70771 & 9.41 \\
& & & & & & \\
\hline
\end{tabular}

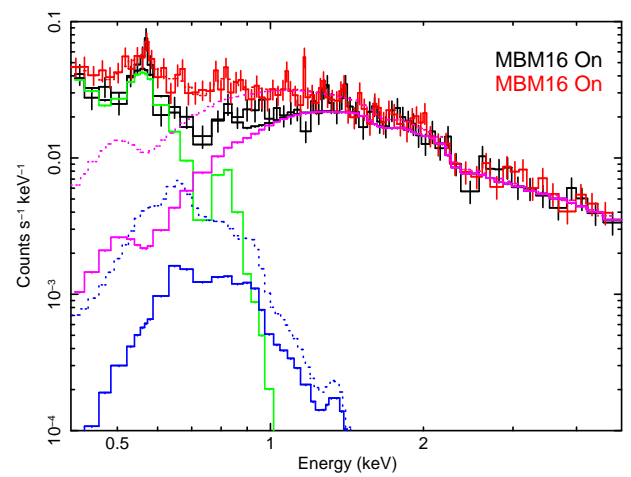

FIG. 1.- The spectra of MBM 16 On- (black) and Off-cloud (red), along with the best fitting model. We show the decomposition of the best fitting model into its various components: the foreground emission (in green), the GH/CGM emission (in blue), and the extragalactic emission from unresolved point sources (in magenta). The solid lines are for the On-cloud, and the dotted lines are for Off-cloud.

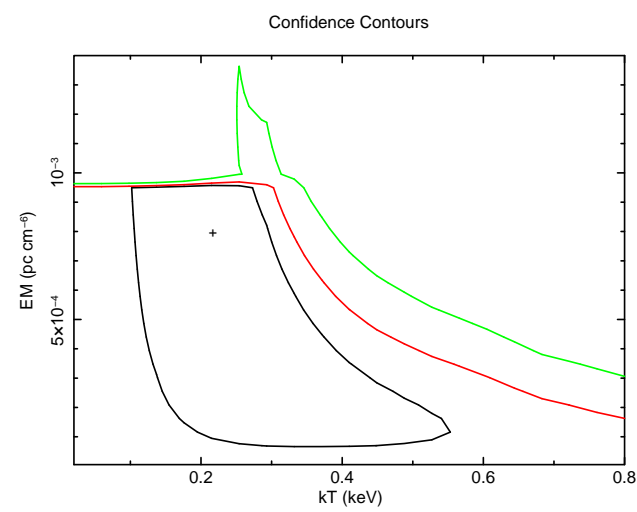

FIG. 2.- The GH temperature and emission measure constraints at the $68 \%$ (black), 90\% (red), and 99\% (green) confidence levels from the simultaneously fitting of MBM 16 On- and Off-cloud.

GH/CGM component is negligible comparing to other components (foreground and extragalactic background) as seen in Figure 1 .

In fact, the magnitude of the GH/CGM component is consistent with zero. Figure 2 shows the statistical relevance of the GH/CGM component as function of temperature and emission measure. The contour plot shows the $68 \%, 90 \%$, and $99 \%$ confidence region as black, red, and green lines respectively. At $90 \%$ confidence levels, the upper limit of emission measure is $\sim 9 \times 10^{-4}$ pc $\mathrm{cm}^{-6}$, while the lower limit is well consistent with zero. We tested the case with zero contribution from the GH/CGM component in our model. From the f-test, there is a zero probability significance of improving the fit by including a GH/CGM contribution. Our results are therefore consistent with no GH/CGM emission in the direction of MBM16.

We also verified the effect of the foreground model on the statistical significance of our model. For example, we used the AtomDB Change Exchange (ACX, Smith et al. (2012)) model to represent the SWCX component, instead of the Gaussian lines. We found that the model used to represent the foreground does not affect our result, and all our tests are consistent with a negligible contribution from GH/CGM.

\section{DISCUSSION}

Multiple authors have investigated the X-ray emission of empty fields in the sky to extrapolate the contribution from GH/CGM. Those papers show significant variation between pointings, including fields where no significant emission was detected, perhaps an indication of a patchy medium. However, those results are significantly affected by uncertainty in the removal of background emission, making any strong conclusion difficult.

The current result shows, without significant systematic uncertainty, that the GH/CGM emission in the direction of MBM 16 is quite small or inexistent, with an upper limit on the plasma emission measure at the lowest end of the sample covered by Henley \& Shelton (2013, Figure 6). Using simple assumptions, this result can be converted to significant upper limits on the density of the medium, depending on the model used. Specifically, assuming a uniform halo, the $90 \%$ C.L. upper limit of $9.5 \times 10^{-4} \mathrm{pc} \mathrm{cm}^{-6}$ for the emission measure would translate to a maximum density of $9.7 \times 10^{-5}\left(Z / Z_{\text {solar }}\right)^{-1} \mathrm{~cm}^{-3}$ for an extended $(100 \mathrm{kpc})$ halo, and $4.4 \times 10^{-4}\left(Z / Z_{\text {solar }}\right)^{-1} \mathrm{~cm}^{-3}$ for a more compact $(5 \mathrm{kpc})$ one. While the statistical uncertainty on this result is still not sufficient to discriminate between GH/CGM models, it places the upper limit on emission measure at the lower end of prediction from uniform halo models, confirming the possibility of a non-uniform halo.

The number of shadow observations currently available is very limited, making any general conclusion limited at this time. A summary of such investigations is available in (Gupta et al. 2009). However, three observed shadow targets are closely spaced together (MBM16, MBM12, and MBM20 see Figure 3) and can be compared directly. Of the three, only MBM16 does not show any GH/CGM emission, which would indicate a highly inhomogeneous medium. However, a closer look at the measurements in the direction of MBM12 and MBM20 may show a different picture. MBM12 (observed by Smith et al. (2007)) is very close to MBM16, and any significant difference between the halo emission in the two directions would be strong evidence of a highly inhomogenous medium. However, Koutroumpa et al. (2011) has shown that the MBM12 observation was affected by strong variation in 


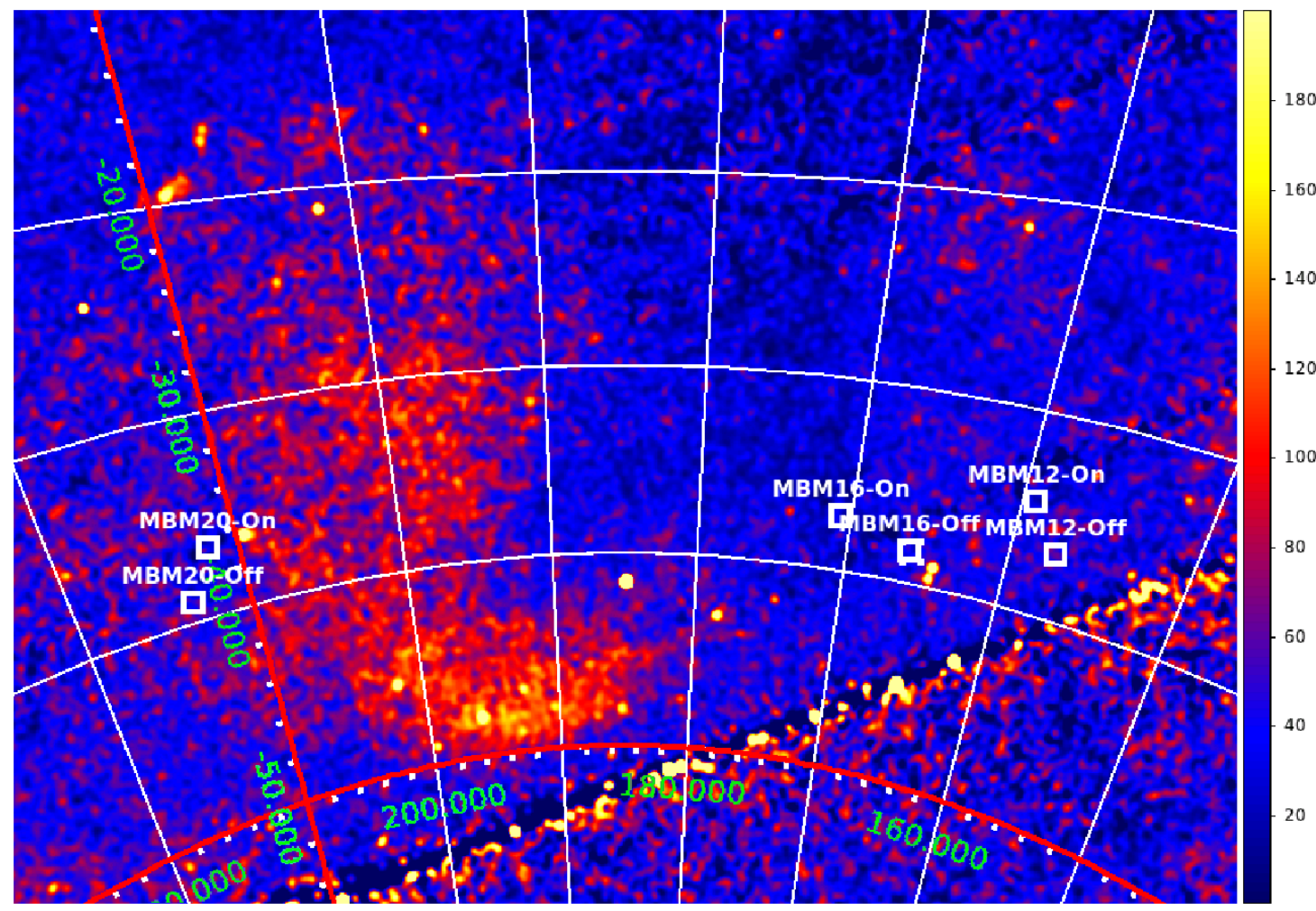

FIG. 3.- The squares $\left(1^{\circ} \times 1^{\circ}\right.$, for plotting purpose) show locations of three shadow targets, MBM 12, MBM 16, and MBM 20, in ROSAT All-sky Survey map in R4 band (units $10^{-6}$ counts s$^{-1} \operatorname{arcmin}^{-2}$, the grid is in Galactic coordinate).

TABLE 2

Spectral Fitting Result

\begin{tabular}{|c|c|c|c|c|c|c|c|c|c|c|}
\hline \multicolumn{2}{|c|}{ LHB } & \multicolumn{3}{|c|}{ SWCX } & \multicolumn{2}{|r|}{$\mathrm{GH}$} & \multicolumn{2}{|c|}{ Power Law } & \multirow[b]{2}{*}{$\begin{array}{c}\text { O I } \\
(\mathrm{LU})\end{array}$} & \multirow[t]{2}{*}{$\chi^{2} /$ dof } \\
\hline $\begin{array}{c}\mathrm{T} \\
\mathrm{keV}\end{array}$ & $\begin{array}{c}\mathrm{EM} \\
\text { pc } \mathrm{cm}^{-6}\end{array}$ & $\begin{array}{l}\text { O VII } \\
(\mathrm{LU})\end{array}$ & $\begin{array}{l}\mathrm{O} \mathrm{VIIII}^{\mathrm{a}} \\
(\mathrm{LU})\end{array}$ & $\begin{array}{c}\text { O VIII' } \\
(\mathrm{LU})\end{array}$ & $\begin{array}{c}\mathrm{T} \\
\mathrm{keV}\end{array}$ & $\begin{array}{l}\mathrm{EM} \\
10^{-3} \mathrm{pc} \mathrm{cm}-6\end{array}$ & $\Gamma$ & Norm $^{\mathrm{c}}$ & & \\
\hline $0.09_{+0.03}^{-0.04}$ & $0.03_{+0.12}^{-0.01}$ & $3.78_{+0.80}^{-0.87}$ & $0.86_{+0.40}^{-0.39}$ & $0.34_{+0.19}^{-0.20}$ & $0.22_{+0.44}^{-0.20}$ & $\begin{array}{c}0.79_{+0.18}^{-0.79} \\
\end{array}$ & $\begin{array}{r}1.58_{+0.08}^{-0.08}\end{array}$ & $1.03_{+0.06}^{-0.06}$ & $0.83_{+0.65}^{-0.83}$ & $302.61 / 326$ \\
\hline
\end{tabular}

Note. - Errors are quoted at $90 \%$ confidence.

${ }^{\text {a }} \mathrm{O}$ VIII line at $0.57 \mathrm{keV}$.

b $\mathrm{O}$ VIII line at $0.81 \mathrm{keV}$.

c Normalization of power law fit at $1 \mathrm{keV}$ in units of $10^{-3}$ photons $\mathrm{keV}^{-1} \mathrm{~s}^{-1} \mathrm{~cm}^{-2}$.

SWCX between on and off-cloud, making any conclusion impossible. It is conceivable, indeed, that the measured $\mathrm{GH} / \mathrm{CGM}$ emission measured in the direction of MBM12 was, in fact, the result of SWCX, and that the GH/CGM emission was compatible with MBM16.

MBM20 is slightly further away, and its positive emission would point to a variation in GH/CGM on the scale of some degrees. However, MBM20 is at the edge of the
Eridanus enhancement (Burrows et al. 1993, Snowden et al. 1995), which again could mimic any emission from GH/CGM. In conclusion, the current, very limited evidence, is in fact consistent with a region of very low, or non-existent emission from GH/CGM.

This work was funded by NASA grant NNX11AF80G and NNX13AI04G.

\section{REFERENCES}

Anders, E. \& Grevesse, N. 1989, Geochim. Cosmochim. Acta, 53, 197

Arnaud, K. A. 1996, in ASP Conf. Ser. 101: Astronomical Data Analysis Software and Systems V, Vol. 5, 17

Boulanger, F. \& Perault, M. 1988, ApJ, 330, 964

Burrows, D. N. \& Mendenhall, J. A. 1991, Nature, 351, 629

Burrows, D. N., Singh, K. P., Nousek, J. A., Garmire, G. P., \& Good, J. 1993, ApJ, 406, 97

Cen, R. \& Ostriker, J. P. 1999, ApJ, 519, L109

Dickey, J. M. \& Lockman, F. J. 1990, ARA\&A, 28, 215
Foster, A. R., Ji, L., Smith, R. K., \& Brickhouse, N. S. 2012 ApJ, 756, 128

Galeazzi, M., Gupta, A., Covey, K., \& Ursino, E. 2007, ApJ, 658, 1081

Gupta, A., Galeazzi, M., Koutroumpa, D., Smith, R., \&

Lallement, R. 2009, ApJ, 707, 644

Henley, D. B. \& Shelton, R. L. 2008, ApJ, 676, 335

-. 2010, ApJS, 187, 388

—. 2012, ApJS, 202, 14

—. 2013, ApJ, 773, 92 
Kalberla, P. M. W., Burton, W. B., Hartmann, D., Arnal, E. M., Bajaja, E., Morras, R., \& Pöppel, W. G. L. 2005, A\&A, 440, 775

Koutroumpa, D., Smith, R. K., Edgar, R. J., Kuntz, K. D., Plucinsky, P. P., \& Snowden, S. L. 2011, ApJ, 726, 91

Kuntz, K. D. \& Snowden, S. L. 2000, ApJ, 543, 195

Liu, W., Galeazzi, M. Uprety, Y., Ursino, E., Koutroumpa, D., Kuntz, K. D., McCammon, D., \& Snowden, S. L. 2015, in preparation

Miller, M. J. \& Bregman, J. N. 2015, ApJ, 800, 14

Mitsuishi, I., Gupta, A., Yamasaki, N. Y., Takei, Y., Ohashi, T., Sato, K., Galeazzi, M., Henry, J. P., \& Kelley, R. L. 2012, PASJ, 64, 18

Nicastro, F., Zezas, A., Drake, J., Elvis, M., Fiore, F., Fruscione, A., Marengo, M., Mathur, S., \& Bianchi, S. 2002, ApJ, 573, 157

Sekiya, N., Yamasaki, N. Y., Mitsuda, K., \& Takei, Y. 2014 PASJ, 66, L3
Smith, R. K., Bautz, M. W., Edgar, R. J., Fujimoto, R.,

Hamaguchi, K Hughes, J. P Ishida, M Kelley, R

Kilbourne, C. A., Kuntz, K. D., McCammon, D., Miller, E.,

Mitsuda, K., Mukai, K., Plucinsky, P. P., Porter, F. S.,

Snowden, S. L., Takei, Y., Terada, Y., Tsuboi, Y., \& Yamasaki, N. Y. 2007, PASJ, 59, 141

Smith, R. K., Edgar, R. J., Plucinsky, P. P., Wargelin, B. J., Freeman, P. E., \& Biller, B. A. 2005, ApJ, 623, 225

Smith, R. K., Foster, A. R., \& Brickhouse, N. S. 2012 Astronomische Nachrichten, 333, 301

Snowden, S. L., Burrows, D. N., Sanders, W. T., Aschenbach, B., \& Pfeffermann, E. 1995, ApJ, 439, 399

Snowden, S. L., Egger, R., Finkbeiner, D. P., Freyberg, M. J., \& Plucinsky, P. P. 1998, ApJ, 493, 715

Snowden, S. L., Hasinger, G., Jahoda, K., Lockman, F. J., McCammon, D., \& Sanders, W. T. 1994, ApJ, 430, 601

Snowden, S. L., Mebold, U., Hirth, W., Herbstmeier, U., \& Schmitt, J. H. M. 1991, Science, 252, 1529

Williams, R. J., Mathur, S., Nicastro, F., \& Elvis, M. 2007, ApJ, 665,247

Yoshino, T., Mitsuda, K., Yamasaki, N. Y., Takei, Y., Hagihara T., Masui, K., Bauer, M., McCammon, D., Fujimoto, R., Wang, Q. D., \& Yao, Y. 2009, PASJ, 61, 805 